\title{
A validation of the diathesis-stress model for depression in Generation Scotland
}

\author{
Aleix Arnau-Soler $\mathbb{D}^{1}$, Mark J. Adams $\mathbb{E}^{2}$, Toni-Kim Clarke², Donald J. Maclntyre $\mathbb{E}^{2}$, Keith Milburn ${ }^{3}$, Lauren Navrady $\mathbb{E}^{2}$, \\ Generation Scotland, Major Depressive Disorder Working Group of the Psychiatric Genomics Consortium, \\ Caroline Hayward (10), Andrew Mclntosh (D) $^{2,5}$ and Pippa A. Thomson (D)
}

\begin{abstract}
Depression has well-established influences from genetic and environmental risk factors. This has led to the diathesisstress theory, which assumes a multiplicative gene-by-environment interaction (GXE) effect on risk. Recently, ColodroConde et al. empirically tested this theory, using the polygenic risk score for major depressive disorder (PRS, genes) and stressful life events (SLE, environment) effects on depressive symptoms, identifying significant GxE effects with an additive contribution to liability. We have tested the diathesis-stress theory on an independent sample of 4919 individuals. We identified nominally significant positive GxE effects in the full cohort $\left(R^{2}=0.08 \%, p=0.049\right)$ and in women $\left(R^{2}=0.19 \%, p=0.017\right)$, but not in men $\left(R^{2}=0.15 \%, p=0.07\right)$. GxE effects were nominally significant, but only in women, when SLE were split into those in which the respondent plays an active or passive role $\left(R^{2}=0.15 \%, p=\right.$ $0.038 ; R^{2}=0.16 \%, p=0.033$, respectively). High PRS increased the risk of depression in participants reporting high numbers of SLE $\left(p=2.86 \times 10^{-4}\right)$. However, in those participants who reported no recent SLE, a higher PRS appeared to increase the risk of depressive symptoms in men $(\beta=0.082, p=0.016)$ but had a protective effect in women $(\beta=$ $-0.061, p=0.037)$. This difference was nominally significant $(p=0.017)$. Our study reinforces the evidence of additional risk in the aetiology of depression due to GxE effects. However, larger sample sizes are required to robustly validate these findings.
\end{abstract}

\section{Introduction}

Stressful life events (SLE) have been consistently recognized as a determinant of depressive symptoms, with many studies reporting significant associations between SLE and major depressive disorder $(\mathrm{MDD})^{1-7}$. Some studies suggest that severe adversity is present before the onset of illness in over $50 \%$ of individuals with depression ${ }^{8}$ and may characterize a subtype of cases ${ }^{9}$. However, some individuals facing severe stress never present symptoms of depression ${ }^{10}$. This has led to a suggestion that the

\footnotetext{
Correspondence: Aleix Arnau-Soler (aleix.arnau.soler@igmm.ed.ac.uk) or Pippa A. Thomson (Pippa.Thomson@ed.ac.uk)

${ }^{1}$ Medical Genetics Section, Centre for Genomic and Experimental Medicine and MRC Institute of Genetics and Molecular Medicine, University of Edinburgh, Edinburgh, UK

${ }^{2}$ Division of Psychiatry, Deanery of Clinical Sciences, Royal Edinburgh Hospital, University of Edinburgh, Morningside Park, Edinburgh EH10 5HF, UK

Full list of author information is available at the end of the article.
}

interaction between stress and an individual's vulnerability, or diathesis, is a key element in the development of depressive symptoms. Such vulnerability can be conceived as a set of biological factors that predispose to illness. Several diathesis-stress models have been successfully applied across many psychopathologies ${ }^{11-15}$.

The diathesis-stress model proposes that a latent diathesis may be activated by stress before psychopathological symptoms manifest. Some levels of diathesis to illness are present in everybody, with a threshold over which symptoms will appear. Exceeding such a threshold depends on the interaction between diathesis and the degree of adversity faced in SLE, which increases the liability to depression beyond the combined additive effects of the diathesis and stress alone ${ }^{11}$. Genetic risk factors can, therefore, be conceived as a genetic diathesis. Thus, this genetically driven effect produced by the diathesis-

\section{(c) The Author(s) 2019}

(c) (i) Open Access This article is licensed under a Creative Commons Attribution 4.0 International License, which permits use, sharing, adaptation, distribution and reproduction in any medium or format, as long as you give appropriate credit to the original author(s) and the source, provide a link to the Creative Commons license, and indicate if changes were made. The images or other third party material in this article are included in the article's Creative Commons license, unless indicated otherwise in a credit line to the material. If material is not included in the article's Creative Commons license and your intended use is not permitted by statutory regulation or exceeds the permitted use, you will need to obtain permission directly from the copyright holder. To view a copy of this license, visit http://creativecommons.org/licenses/by/4.0/. 
stress interaction can be seen as a gene-by-environment interaction $(\mathrm{GxE})$.

MDD is characterized by a highly polygenic architecture, composed of common variants with small effect and/or rare variants ${ }^{16}$. Therefore, interactions in depression are also expected to be highly polygenic. In recent years, with the increasing success of genome-wide association studies, GxE studies in depression have shifted towards hypothesis-free genome-wide and polygenic approaches that capture liability to depression using genetic data ${ }^{17-23,24}$. Recent advances in genomics and the massive effort from national institutions to collect genetic, clinical and environmental data on large population-based samples now provide an opportunity to empirically test the diathesis-stress model for depression. The construction of polygenic risk scores (PRS) offers a novel paradigm to quantify genetic diathesis into a single genetic measure, allowing us to study $\mathrm{GxE}$ effects with more predictive power than any single variant ${ }^{25-28}$. PRS are genetic indicators of the aggregated number of risk alleles carried by an individual weighted by their allelic effect estimated from genome-wide association studies. This polygenic approach to assessing the diathesis-stress model for depression has been tested using either childhood trauma ${ }^{17,19,24}$ or adult SLE $^{18,23,24}$ as measures of environmental adversity.

Recently, Colodro-Conde et al. ${ }^{23}$ provided a direct test of the diathesis-stress model for recent SLE and depressive symptoms. In this study, Colodro-Conde et al. used PRS weighted by the most recent genome-wide meta-analysis conducted by the Psychiatric Genetics Consortium (PGC; $N=159,601$ ), and measures of three environmental exposures: lack of social support, "personal" SLE, and "network" SLE. Colodro-Conde et al. reported a significant additive risk on liability to depression due to a GxE effect in individuals who combine a high genetic predisposition to MDD and a high number of reported "personal" SLE, mainly driven by effects in women. A significant effect of interaction was not detected in males. They found no significant interaction between the genetic diathesis and "network" SLE or social support. They concluded that the effect of stress on risk of depression was dependent on an individual's diathesis, thus supporting the diathesis-stress theory. In addition, they suggested possible sex-specific differences in the aetiology of depression. However, Colodro-Conde et al. findings have not, to our knowledge, been independently validated.

In the present study, we aim to test the diathesis-stress model in an independent sample of 4919 unrelated white British participants from a further longitudinal follow-up from Generation Scotland, and assess the differences between women and men, using self-reported depressive symptoms and recent SLE.

\section{Materials and methods \\ Sample description}

Generation Scotland is a family-based population cohort recruited throughout Scotland by a crossdisciplinary collaboration of Scottish medical schools and the National Health Service (NHS) between 2006 and $2011^{29}$. At baseline, blood and salivary DNA samples from Generation Scotland participants were collected, stored and genotyped at the Wellcome Trust Clinical Research Facility, Edinburgh. Genome-wide genotype data were generated using the Illumina HumanOmniExpressExome-8 v1.0 DNA Analysis BeadChip (San Diego, CA, USA) and Infinium chemistry ${ }^{30}$. The procedures and further details for DNA extraction and genotyping have been extensively described elsewhere $^{31,32}$. In 2014, 21,525 participants from Generation Scotland eligible for re-contact were sent self-reported questionnaires as part of a further longitudinal assessment funded by a Wellcome Trust Strategic Award "STratifying Resilience and Depression Longitudinally" (STRADL) ${ }^{33}$ to collect new and updated mental health questionnaires including psychiatric symptoms and SLE measures. 9618 re-contacted participants from Generation Scotland agreed to provide new measures to the mental health follow-up $^{33}$ (44.7\% response rate). Duplicate samples, those showing sex discrepancies with phenotypic data, or that had more than $2 \%$ missing genotype data, were removed from the sample, as were samples identified as population outliers in principal component analysis (mainly non-Caucasians and Italian ancestry subgroups). In addition, individuals with diagnoses of bipolar disorder, or with missing SLE data, were excluded from the analyses. SNPs with more than $2 \%$ of genotypes missing, Hardy-Weinberg Equilibrium test $p<1 \times 10^{-6}$, or a minor allele frequency lower than $1 \%$, were excluded. Individuals were then filtered by degree of relatedness (pi-hat $<0.05$ ) using PLINK v1.9 $9^{34}$, maximizing retention of those participants reporting higher numbers of SLE (see phenotype assessment below). After quality control, the final dataset comprised 4919 unrelated individuals of European ancestry and 560351 SNPs (mean age at questionnaire: 57.2 , s.d. $=12.2$, range $22-95$; women: $n=2990-60.8 \%$, mean age 56.1, s.d. $=12.4$; men: $n=1$ 929-39.2\%, mean age 58.7 , s.d. $=11.8$ ). Further details on the recruitment procedure and Generation Scotland profile are described in detail elsewhere ${ }^{29,31,35-37}$. All participants provided written consent. All components of Generation Scotland and STRADL obtained ethical approval from the Tayside Committee on Medical Research Ethics on behalf of the National Health Service (reference 05/s1401/89). Generation Scotland data is available to researchers on application to the Generation Scotland Access Committee (access@generationscotland.org). 


\section{Phenotype assessment}

Participant self-reported current depressive symptoms through the 28-item scaled version of The General Health Questionnaire $^{38,39}$. The General Health Questionnaire is a reliable and validated psychometric screening tool to detect common psychiatric and non-psychotic conditions (General Health Questionnaire Cronbach alpha coefficient: $0.82-0.86)^{40}$. This consists of 28 items designed to identify whether an individual's current mental state has changed over the last 2 weeks from their typical state. The questionnaire captures core symptoms of depression through subscales for severe depression, emotional (e.g., anxiety and social dysfunction) and somatic symptoms linked to depression. These subscales are highly correlated ${ }^{41}$ and suggest an overall general factor of depres$\operatorname{sion}^{42}$. Participants rated the 28 items on a four-point Likert scale from 0 to 3 to assess its degree or severity ${ }^{40}$ (e.g., Have you recently felt that life is entirely hopeless? "Not at all", "No more than usual", "Rather more than usual", "Much more than usual"), resulting on an 84-point scale depression score. The Likert scale, which provides a wider and smoother distribution ${ }^{40}$, may be more sensitive to detect changes in mental status in those participants with chronic conditions or chronic stress who may feel their current symptoms as "usual" chopathology changes as response to stress. The final depression score was log transformed to reduce the effect of positive skew and provide a better approximation to a normal distribution. In addition, participants completed the Composite International Diagnostic Interview-Short Form, which diagnoses lifetime history of MDD according to DSM-IV criteria ${ }^{44}$. The depression score predicted lifetime history of MDD (odd ratio $=1.91,95 \%$ confidence intervals $1.80-2.02, p=1.55 \times 10^{-102}, N=8994$ ), with a 3.8-fold increased odds of having a lifetime history of MDD between participants in the top and bottom deciles, thus supporting the usefulness of the depression score in understanding MDD. To improve interpretation, we scaled the depression score to a mean of 0 when required (Fig. 3).

Data from a self-reported questionnaire based on the List of Threating Experiences ${ }^{45}$ was used to construct a measure of common SLE over the previous 6 months. The List of Threatening Experiences is a reliable psychometric device to measure psychological "stress" ${ }^{46,47}$. It consists of a 12-item questionnaire to assess SLE with considerable long-term contextual effects (e.g., Over last 6 months, did you have a serious problem with a close friend, neighbor or relatives?). A final score reflecting the total number of SLE (TSLE) ranging from 0 to 12 was constructed by summing the "yes" responses. Additionally, TSLE was split into two categories based on those items measuring SLE in which the individual may play and active role exposure to SLE, and therefore in which the SLE is influenced by genetic factors and thus subject to be "dependent" on an individual's own behavior or symptoms (DSLE; 6 items, e.g., $a$ serious problem with a close friend, neighbor or relatives may be subject to a respondent's own behavior), or SLE that are not influenced by genetic factors, likely to be "independent" on a participant's own behavior (ISLE; 5 items, e.g., a serious illness, injury or assault happening to a close relative is potentially independent of a respondent's own behavior) ${ }^{45,48}$. The item "Did you/your wife or partner give birth?" was excluded from this categorization. In addition, SLE reported were categorized to investigate the diathesis effect at different levels of exposure, including a group to test the diathesis effect when SLE is not reported. Three levels of SLE reported were defined (0 SLE = "none", 1 or 2 SLE = "low", and 3 or more SLE = "high") to retain a large enough sample size for each group to allow meaningful statistical comparison.

\section{Polygenic profiling and statistical analysis}

Polygenic risk scores (PRS) were generated by PRSice ${ }^{49}$, whose functionality relies mostly on PLINK v1.9 $9^{34}$, and were calculated using the genotype data of Generation Scotland participants (i.e., target sample) and summary statistics for MDD from the PGC-MDD2 GWAS release (July 2016, discovery sample) used by Colodro-Conde et $\mathrm{al}^{23}$, with the added contribution from QIMR cohort and the exclusion of Generation Scotland participants, resulting in summary statistics for MDD derived from a sample of 50,455 cases and 105,411 controls.

Briefly, PRSice removed strand-ambiguous SNPs and clump-based pruned $\left(r^{2}=0.1\right.$, within a $10 \mathrm{Mb}$ window $)$ our target sample to obtain the most significant independent SNPs in approximate linkage equilibrium. Independent risk alleles were then weighted by the allelic effect sizes estimated in the independent discovery sample and aggregated into PRS. PRS were generated for eight $p$ thresholds ( $p$ thresholds: $<5 \times 10^{-8},<1 \times 10^{-5},<0.001,<$ $0.01,<0.05,<0.1,<0.5,<=1)$ determined by the discovery sample and standardized (See Supplementary Table 1 for summary of PRS).

A genetic relationship matrix (GRM) was calculated for each dataset (i.e., full cohort, women, and men) using GCTA $1.26 .0^{50}$. Mixed linear models using the GRM were used to estimate the variance in depression score explained by PRS, SLEs and their interaction; and stratified by sex. Twenty principal components were calculated for the datasets.

The mixed linear model used to assess the effects of PRS is as follows:

$$
\text { Depression }=\beta_{0}+\beta_{1} P R S+G R M+\text { Covariates }
$$


Mixed linear models used to assess the effect of the stressors are as follows:

$$
\begin{aligned}
& \text { Depression }=\beta_{0}+\beta_{1} \text { TSLE }+ \text { GRM }+ \text { Covariates } \\
& \text { Depression }=\beta_{0}+\beta_{1} D S L E+G R M+\text { Covariates } \\
& \text { Depression }=\beta_{0}+\beta_{1} I S L E+G R M+\text { Covariates }
\end{aligned}
$$

Following Colodro-Conde et al. ${ }^{23}$, covariates (i.e., age, age $^{2}$, sex, age-by-sex and age ${ }^{2}$-by-sex interactions, and 20 principal components) were regressed from PRS (PRS') and SLE scores (i.e., TSLE', DSLE' and ISLE'; SLEs') before fitting models in GCTA to guard against confounding influences on the PRS-by-SLEs interactions ${ }^{51}$. PRS' and SLEs' were standardized to a mean of 0 and a standard deviation of 1 . The Mixed linear models (i.e., the diathesis-stress model) used to assess GxE effects are as follows:

$$
\begin{aligned}
& \text { Depression }=\beta_{0}+\beta_{1} P R S^{\prime}+\beta_{2} T S L E^{\prime} \\
& +\beta_{3} P^{\prime} S^{\prime} x T S L E^{\prime}+G R M+\text { Covariates } \\
& \text { Depression }=\beta_{0}+\beta_{1} P R S^{\prime}+\beta_{2} D S L E^{\prime} \\
& +\beta_{3} P R S^{\prime} x D S L E^{\prime}+G R M+\text { Covariates } \\
& \text { Depression }=\beta_{0}+\beta_{1} P R S^{\prime}+\beta_{2} I S L E^{\prime}+\beta_{3} P R S^{\prime} x I S L E^{\prime} \\
& + \text { GRM }+ \text { Covariates }
\end{aligned}
$$

Covariates fitted in the models above were age, age $\mathrm{e}^{2}$, sex, age-by-sex, age ${ }^{2}$-by-sex, and 20 principal components. Sex and its interactions (age-by-sex and age ${ }^{2}$-bysex) were omitted from the covariates when stratifying by sex. All parameters from the models were estimated using GCTA and the significance of the effect $(\beta)$ from fixed effects assessed using a Wald test. The significance of main effects (PRS and SLEs) allowed for nominally testing the significance of interactions at $p$-threshold $=0.05$. To account for multiple testing correction, a Bonferroni's adjustment correcting for 8 PRS and 3 measures of SLE tested (24 tests) was used to establish a robust threshold for significance at $p=2.08 \times 10^{-3}$.

The PRS effect on depression score at different levels of exposure was further examined for the detected nominally significant interactions by categorizing participants on three groups based on the number of SLE reported (i.e., "none", "low" or "high"). Using linear regression, we applied a least squares approach to assess PRS' effects on the depression score in each SLE category. Further conservative Bonferroni correction to adjust for the 3 SLE categories tested established a threshold for significance of $p=6.94 \times 10^{-4}$.

Differences on the estimated size of GxE effect between women and men were assessed by comparing a $z$-score to the standard normal distribution ( $\alpha=0.05$, one-tailed). $Z$ scores were derived from $\operatorname{GxE}$ estimates $(\beta)$ and standard errors (SE) detected in women and men as follows:

$$
Z \text {-score }=\frac{\beta_{\text {women }}-\beta_{\text {men }}}{\sqrt{S E\left(\beta_{\text {women }}\right)^{2}+S E\left(\beta_{\text {men }}\right)^{2}}}
$$

\section{Results}

PRS for MDD significantly predicted the depression score across the whole sample $(\beta=0.080$, s.e. $=0.014$, $p=7.53 \times 10^{-9}$ ) explaining $0.64 \%$ of the variance at its best $p$-threshold ( $p$-threshold $=0.1$; Fig. 1a). Stratifying by sex, PRS significantly predicted the depression score in both sexes, explaining $0.59 \%$ in men and $0.67 \%$ in women (men: $p$-threshold $=0.1, \beta=0.077$, s.e. $=0.022$, $p=2.09 \times 10^{-4}$; women: $p$-threshold $=0.1, \beta=0.082$, s. e. $=0.018, p=4.93 \times 10^{-6}$; Fig. 1a). Self-reported SLE over the last 6 months (TSLE, mean $=1.3 \mathrm{SLE}$, s.d. $=$ 1.5) also significantly predicted depression score for the whole sample and stratified by sex (full cohort: variance explained $=4.91 \%, \beta=0.222$, s.e. $=0.014, p=9.98 \times$ $10^{-59}$; men: $4.19 \%, \beta=0.205$, s.e. $=0.021, p=2.23 \times$ $10^{-22}$; women: $5.33 \%, \beta=0.231$, s.e. $=0.018, p=7.48 \times$ $10^{-38}$; Fig. 1b). Overall, significant additive contributions from genetics and SLE to depression score were detected in all participants and across sexes. There was no significant difference in the direct effect of TSLE between women and men $(p=0.17)$. However, the variance in depression score explained by the TSLE appeared to be lower than the variance explained by the measure of personal SLE (PSLE) used in ColodroConde et al..$^{23}(12.9 \%)$. This may, in part, be explained by different contributions of dependent and independent SLE items screened in Colodro-Conde et al. compared to our study. Although questions about dependent SLE (DSLE, mean $=0.4$ SLE) represented over $28 \%$ of the TSLE-items reported in our study, the main effect of DSLE explained approximately $93 \%$ of the amount of variance explained by TSLE (full cohort: variance explained $=4.56 \%, \beta=0.212$, s.e. $=0.014, p=$ $1.73 \times 10^{-54}$; men: $3.74 \%, \beta=0.193$, s.e. $=0.021, p=$ $9.66 \times 10^{-21}$; women: $5.07 \%, \beta=0.225$, s.e. $=0.018, p=$ $8.09 \times 10^{-35}$; Fig. 1b). Independent SLE (ISLE, mean $=$ $0.85 \mathrm{SLE}$ ), which represented over $69 \%$ of TSLE-items, explained approximately $57 \%$ of the amount of variance explained by TSLE (full cohort: variance explained $=$ $2.80 \%, \beta=0.167$, s.e. $=0.014, p=1.32 \times 10^{-33}$; men: $2.44 \%, \beta=0.156$, s.e. $=0.022, p=2.88 \times 10^{-13}$; women: $3.02 \%, \beta=0.174$, s.e. $=0.018, p=5.20 \times 10^{-22}$; Fig. $1 \mathrm{~b}$ ). To explore the contribution from each measure, we combined DSLE and ISLE together in a single model. DSLE explained $3.34 \%$ of the variance in depression score compared to $1.45 \%$ of the variance being 


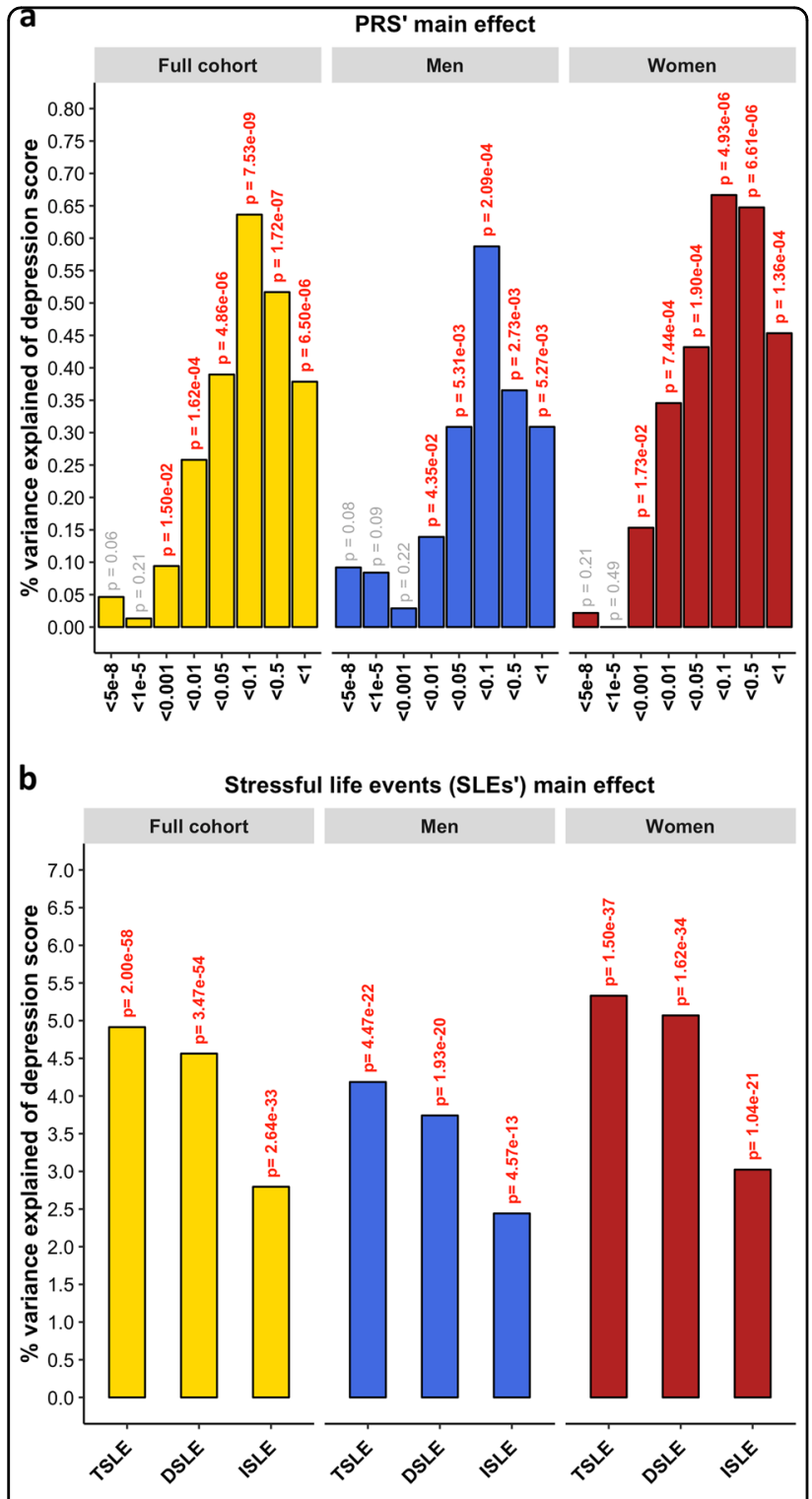

Fig. 1 Prediction of depression symptoms and SLE using the PRS for MDD. a Association between polygenic risk scores (PRS) and depression score (main effects, one-sided tests). PRS were generated at 8 -threshold levels using summary statistics from the Psychiatric Genetic Consortium MDD GWAS (released July 2016) with the exclusion of Generation Scotland participants. The depression score was derived from The General Health Questionnaire. The $Y$-axis represents the \% of variance of depression score explained by PRS main effects. The full cohort (yellow) was split into men (blue) and women (red). In Colodro-Conde et al. PRS for MDD significantly explained up to $0.46 \%$ of depression score in their sample $(\sim 0.39 \%$ in women and $\sim 0.70 \%$ in men). $\mathbf{b}$ Association between reported number of SLE and depression score (main effect, one-sided tests, results expressed in \% of variance in depression score explained). SLE were self-reported through a brief life-events questionnaire based on the List of Threatening Experiences and categorized into: total number of SLE reported (TSLE), "dependent" SLE (DSLE) or "independent" SLE (ISLE). The full cohort (yellow) was split into men (blue) and women (red). In Colodro-Conde et al. "personal" SLE significantly explained up to $12.9 \%$ of depression score variance in their sample $(\sim 11.5 \%$ in women and $\sim 16 \%$ in men) ${ }^{23}$ explained by ISLE, suggesting that DSLE have a greater effect on liability to depressive symptoms than ISLE.

A diathesis-stress model for depression was tested to assess GxE effects. We detected significant, albeit weak, GxE effects on depression score (Fig. 2). The PRS interaction with TSLE was nominally significant in the full cohort $\left(\beta=0.028\right.$, s.e. $\left.=0.014, R^{2}=0.08 \%, p=0.049\right)$ and slightly stronger in women $\left(\beta=0.044\right.$, s.e. $=0.018, R^{2}=$ $0.19 \%, p=0.017$; Fig. $2 \mathrm{a}$ ), compared to men in which the effect was not significant $\left(\beta=0.039\right.$, s.e. $=0.022, R^{2}=$ $0.15 \%, p=0.07)$. However, these results did not survive correction for multiple testing $\left(p>2.08 \times 10^{-3}\right)$.

The best-fit threshold was much lower in women $(p$ threshold $=1 \times 10^{-5}$ ) compared to the full sample ( $p$ threshold $=0.01$ ). The size of the GxE effects across sexes at $p$-threshold $=1 \times 10^{-5}$ were significantly different (GxE* $\operatorname{sex} p=0.017$ ), but not at the best $\mathrm{p}$-threshold in the full cohort ( $p$-threshold $=0.01, \mathrm{GxE}^{*} \operatorname{sex} p=0.32$; Fig. 2a). In women, GxE effect with DSLE predicted depression score $\left(p\right.$-threshold $=1 \times 10^{-5} ; \beta=0.039$, s.e. $=0.019, R^{2}$ $=0.15 \%, p=0.038$; Fig. $2 \mathrm{~b}$ and Supplementary Fig. $2 \mathrm{a}$ ), as did the GxE effect with ISLE ( $p$-threshold $=1 \times 10^{-5} ; \beta=$ 0.040 , s.e. $=0.019, R^{2}=0.16 \%, p=0.033$; Fig. $2 \mathrm{c}$ and Supplementary Fig. 2b). No significant interaction was detected in men (best-fit $p$-threshold $=0.1$ ) with either TSLE $\left(\beta=0.039\right.$, s.e. $=0.022, R^{2}=0.15 \%, p=0.072$; Fig. 2a), DSLE $\left(\beta=0.024\right.$, s.e. $=0.022, R^{2}=0.06 \%, p=0.28$; Fig. $2 \mathrm{~b})$ or ISLE $\left(\beta=0.043\right.$, s.e. $=0.022, R^{2}=0.18 \%, p=$ 0.055; Fig. 2c).

To examine these results further and investigate the diathesis effect at different levels of stress, nominally significant GxE were plotted between PRS and categories of SLE (i.e, "none", "low", and "high" SLE reported; Fig. 3). Examining the interaction found in the full cohort (PRS at PGC-MDD GWAS $p$-threshold $=0.01$ ), we detected a significant direct diathesis effect on the risk of depressive symptoms in those participants reporting SLE, with a higher risk when greater numbers of SLE were reported ("low" number of SLE reported: PRS' $\beta=0.043$, s.e. $=$ 0.021, $p=0.039$; "high" number of SLE reported: PRS' $\beta$ $=0.142$, s.e. $=0.039, p=2.86 \times 10^{-4}$; see Table 1 and Fig. $3 a)$. Whereas, in participants who reported no SLE over the preceding 6 months, the risk of depressive symptoms was the same regardless of their diathesis risk ("none" SLE reported: PRS' $\beta=0.021$, s.e. $=0.022, p=0.339$ ). Stratifying these results by sex, we found the same pattern as in the full cohort in women ("none": $p=0.687$; "low": $p=$ 0.023; "high": $p=2 \times 10^{-3}$ ), but not in men ("none": $p=$ 0.307; "low": $p=728$; "high": $p=0.053$; see Table 1 and Fig. 3a). However, the lack of a significant diathesis effect in men may be due to their lower sample size and its corresponding reduced power.

Examining the interaction with PRS at PGC-MDD GWAS $p$-threshold $=1 \times 10^{-5}$, with which a significant 

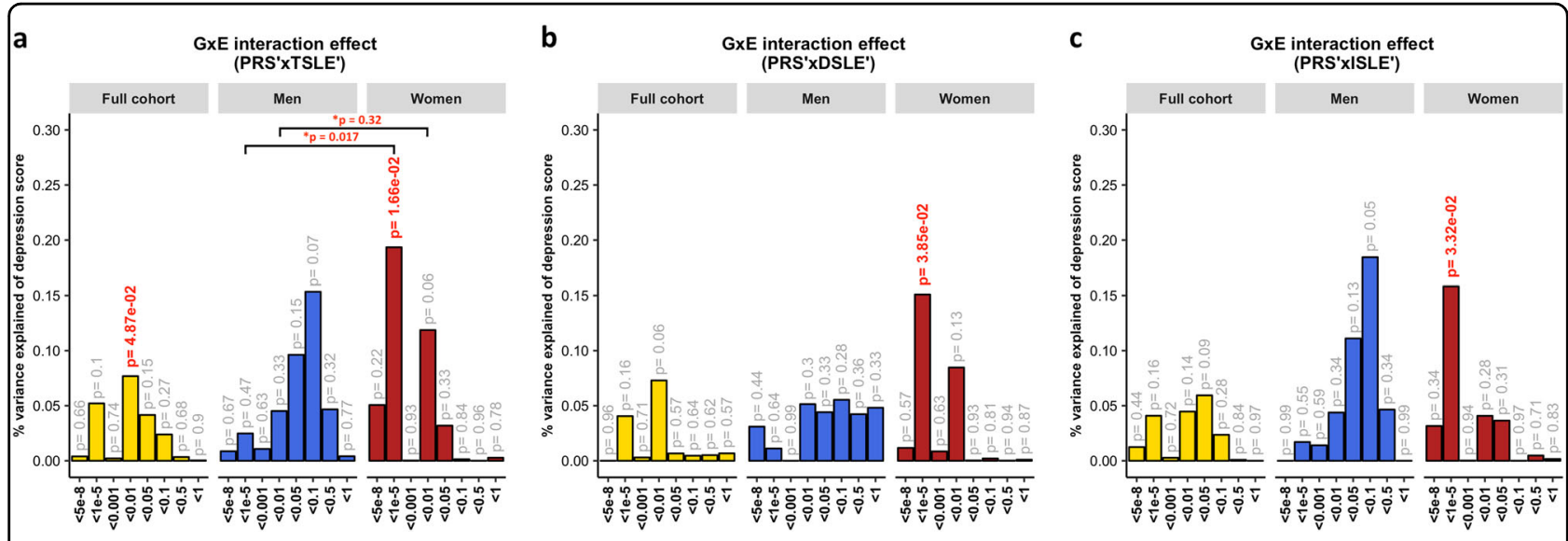

Fig. 2 Association between GxE effect and depression score. The plots show the percentage of depression score explained by the interaction term (two-sided tests) fitted in linear mixed models to empirically test the diathesis-stress model. Red numbers show significant interactions $p$-values. * Shows the significance of the difference in variance explained between sexes. The full cohort (yellow) was split into men (blue) and women (red). PRS were generated at 8 p-threshold levels using summary statistics from the Psychiatric Genetic Consortium MDD GWAS (released July 2016) with the exclusion of Generation Scotland participants. The interaction effect was tested with the number of: (a) SLE (TSLE), (b) "dependent" SLE (DSLE) and c) "independent" SLE (ISLE). In Colodro-Conde et al., the variance of depression score explained in their sample by GxE was $0.12 \%\left(p=7 \times 10^{-3}\right)$. GXE were also significant in women $\left(p=2 \times 10^{-3}\right)$ explaining up to $0.25 \%$ of depression score variation, but not in men $\left(p=0.059 ; R^{2}=0.17 \%\right.$; negative/protective effect on depression score)

interaction was detected in women, we detected a significant diathesis effect on depression score only when stratifying by sex in those participants who did not reported SLE over the last 6 months (see Table 1). The diathesis effect was positive in men (PRS' $\beta=0.082$, s.e. $=$ 0.034, $p=0.016, R^{2}=0.7 \%$; Fig. $3 \mathrm{~b}$ ), consistent with the contribution of risk alleles. Conversely, the diathesis effect was negative in women (PRS' $\beta=-0.061$, s.e. $=0.029, p=$ $0.037, R^{2}=0.4 \%$; Fig. $3 \mathrm{~b}$ ), suggesting a protective effect of increasing PRS in those women reporting no SLE, and consistent with the contribution of alleles to individual sensitivity to both positive and negative environmental effects (i.e., "plasticity alleles" rather than "risk alleles" $)^{52,53}$. This PRS accounted for the effect of just 34 SNPs, and the size of its GxE across sexes were significantly different (GxE* sex $p=0.017$; Fig. $2 \mathrm{a}$ ), supporting possible differences in the underlying stress-response mechanisms between women and men.

\section{Discussion}

The findings reported in this study support those from Colodro-Conde et al. ${ }^{23}$, in an independent sample of similar sample size and study design, and also supports possible sex-specific differences in the effect of genetic risk of MDD in response to SLE.

Both Colodro-Conde et al. and our study suggest that individuals with an inherent genetic predisposition to MDD, reporting high number of recent SLE, are at additional risk of depressive symptoms due to GxE effects, thus validating the diathesis-stress theory. We identified nominally significant $\mathrm{GxE}$ effects in liability to depression at the population level $(p=0.049)$ and in women $(p=$ $0.017)$, but not in men $(p=0.072)$. However, these interactions did not survive multiple testing correction $(p$ $>2.08 \times 10^{-3}$ ) and the power of both studies to draw robust conclusions remains limited ${ }^{54}$. With increased power these studies could determine more accurately both the presence and magnitude of a GxE effect in depression. To better understand the effect of PRS at different levels of exposure to stress, we examined the nominally significant interactions detected in the full sample by categorizing participants on three groups based on the number of SLE reported (i.e., "none", "low" or "high"). We detected a significant diathesis effect on risk of depression only in those participants reporting SLE, but not in those participants that reported no SLE over the preceding 6 months. Furthermore, the diathesis effect was stronger on those participants reporting a "high" number of $\operatorname{SLE}\left(\beta=0.142, p=2.86 \times 10^{-4}\right)$ compared to those participants reporting a "low" number of SLE $(\beta=0.043$, $p=0.039$ ). The former effect was significant and survived a conservative Bonferroni correction to adjust for multiple testing $\left(p<6.94 \times 10^{-4}\right)$. This finding corroborates the diathesis-stress model for depression and supports the results of Colodro-Conde et al. in an independent sample.

To investigate the relative contribution of the GxE to the variance of depression, we examined in the full cohort the total variance of depression score explained by the PRS main effect and the significant GxE effect jointly. Together, they explained $0.34 \%$ of the variance, of which $0.07 \%$ of the variance of the depression score was attributed to the $\mathrm{GxE}$ effect $\left(p\right.$-threshold $=0.01 ;$ PRS $p=1.19 \times 10^{-4}, \operatorname{GxE} p=$ 


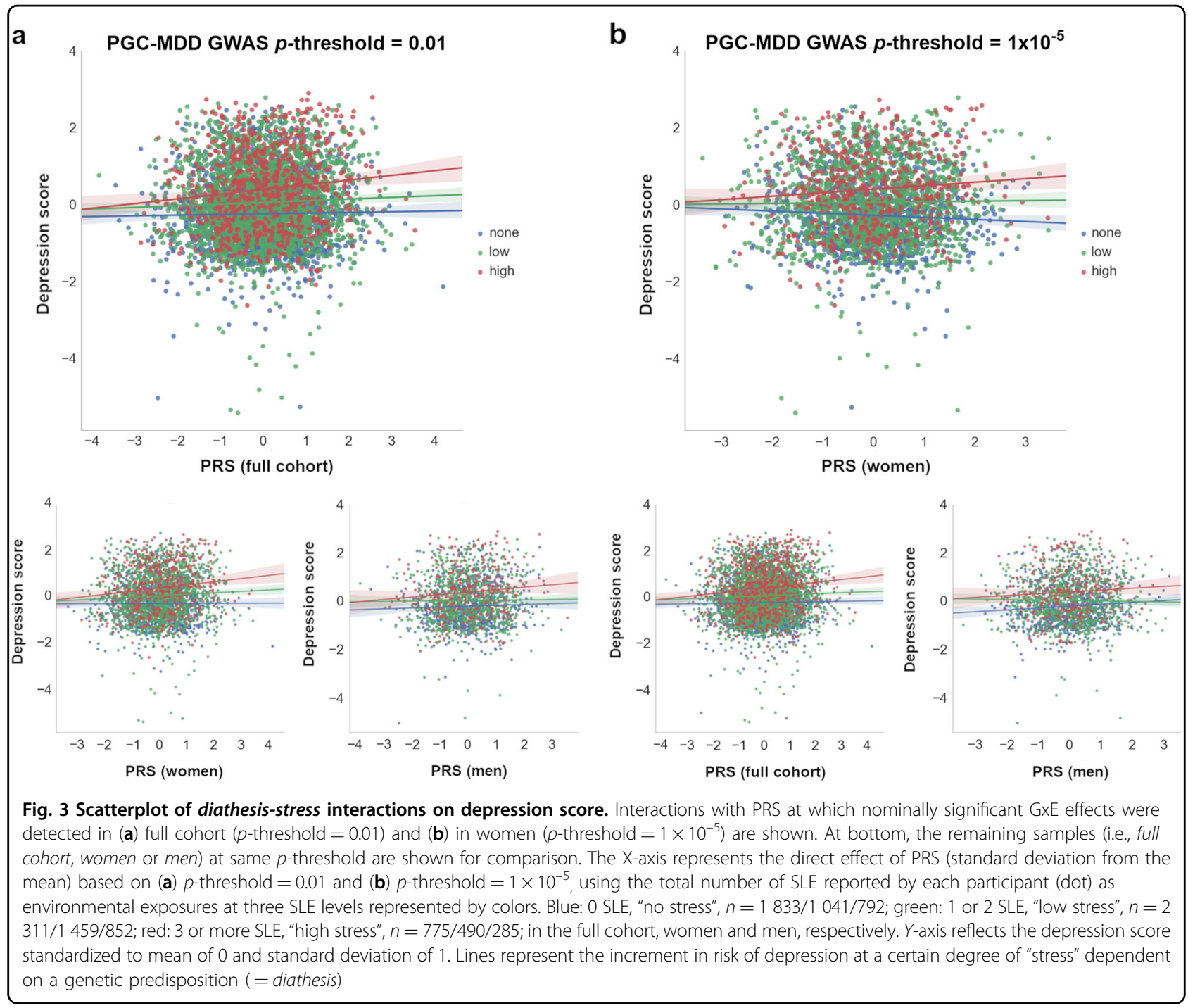

0.049; both derived from the full diathesis-model with TSLE). This is lower than the proportion of variance attributed to common SNPs (8.9\%) in the full PGC-MDD analysis $^{16}$. As Colodro-Conde et al. noted, this result aligns with estimates from experimental organisms suggesting that around $20 \%$ of the heritability may be typically attributed to the effects of $\mathrm{GxE}^{55}$, although it is inconsistent with twin studies of the majority of human traits with the potential exception of depression ${ }^{56}$.

Consistent with PRS predicting "personal" SLE in Colodro-Conde et al., PRS for MDD predicted SLE in our study (see Supplementary Fig. 1), although not at the $p$ threshold at which significant GxE effects were detected. Genetic factors predisposing to MDD may contribute to individuals exposing themselves to, or showing an increased reporting of, SLE via behavioral or personality traits $^{57,58}$. Such genetic mediation of the association between depression and SLE would disclose a gene- environment correlation (i.e., genetic effects on the probability of undergoing a SLE) that hinders interpretion of our findings as pure GxE effects ${ }^{59,60}$. To address this limitation and assess this aspect, following ColodroConde et al., we split the 12-items TSLE measure into SLE that are either potentially "dependent" of a participant's own behavior (DSLE; therefore, potentially driven by genetic factors) or not ("independent" SLE; ISLE) ${ }^{45,48}$. DSLE are reported to be more heritable and have stronger associations with MDD than ISLE ${ }^{48,61,57}$. In our sample, DSLE is significantly heritable $\left(h^{2}{ }_{\mathrm{SNP}}=0.131\right.$, s.e. $=0.071$, $p=0.029)$, supporting a genetic mediation of the association, whereas ISLE is not significantly heritable $\left(h^{2}\right.$ SNP $=0.000$, s.e. $=0.072, p=0.5)^{62}$. Nominally significant GxE effects were seen in women for both DSLE and ISLE, suggesting that both $\mathrm{GxE}$ and gene-environment correlation co-occur. Colodro-Conde et al. did not identify significant GxE using independent SLE as the exposure. 
Table 1 Diathesis effect on depression score in three SLE categories

\begin{tabular}{|c|c|c|c|c|c|c|c|c|c|}
\hline \multirow{2}{*}{$\begin{array}{l}\text { Sample } \\
\text { SLE category }\end{array}$} & \multicolumn{3}{|l|}{ Full cohort ${ }^{a}$} & \multicolumn{3}{|l|}{ Women } & \multicolumn{3}{|l|}{ Men } \\
\hline & None & Low & High & None & Low & High & None & Low & High \\
\hline \multicolumn{10}{|c|}{ PRS at $p$ value threshold $=0.01$} \\
\hline$N$ & 1833 & 2311 & 775 & 1041 & 1459 & 490 & 792 & 852 & 285 \\
\hline Effect & 0.021 & 0.043 & 0.142 & 0.0118 & 0.0617 & 0.1538 & 0.0346 & 0.0113 & 0.1227 \\
\hline s.e. & 0.022 & 0.021 & 0.039 & 0.029 & 0.027 & 0.049 & 0.034 & 0.032 & 0.063 \\
\hline$t$ & 0.957 & 2.07 & 3.644 & 0.403 & 2.274 & 3.112 & 1.021 & 0.348 & 1.947 \\
\hline$p$ value & 0.339 & 0.039 & $2.86 \times 10^{-4}$ & 0.687 & 0.023 & 0.002 & 0.307 & 0.728 & 0.053 \\
\hline Cl (95\%) & $-0.022,0.065$ & $0.002,0.084$ & $0.065,0.218$ & $-0.046,0.069$ & $0.008,0.115 \quad 0$ & $0.057,0.251-$ & $-0.032,0.101$ & $-0.052,0.075$ & $-0.001,0.247$ \\
\hline Sample & \multicolumn{3}{|l|}{ Full cohort } & \multicolumn{3}{|l|}{ Women $^{a}$} & \multicolumn{3}{|l|}{ Men } \\
\hline SLE category & None & Low & High & None & Low & High & None & Low & High \\
\hline \multicolumn{10}{|c|}{ PRS at $\boldsymbol{p}$ value threshold $=1 \times 10^{-\mathbf{5}}$} \\
\hline N & 1833 & 2311 & 775 & 1041 & 1459 & 490 & 792 & 852 & 285 \\
\hline Effect & -0.0022 & 0.0032 & 0.0705 & -0.061 & 0.014 & 0.078 & 0.082 & -0.0176 & 0.0548 \\
\hline s.e. & 0.022 & 0.021 & 0.04 & 0.029 & 0.027 & 0.049 & 0.034 & 0.033 & 0.07 \\
\hline$t$ & -0.098 & 0.153 & 1.76 & -2.086 & 0.541 & 1.609 & 2.416 & -0.537 & 0.778 \\
\hline$p$ value & 0.922 & 0.878 & 0.079 & 0.037 & 0.589 & 0.108 & 0.016 & 0.592 & 0.437 \\
\hline $\mathrm{Cl}(95 \%)$ & $-0.046,0.041$ & $-0.037,0.044$ & $-0.008,0.149$ & $\begin{array}{l}-0.119 \\
-0.004\end{array}$ & $-0.038,0.066$ & $6-0.017,0.174$ & $\begin{array}{ll}74 & 0.015 \\
& 0.149\end{array}$ & $-0.082,0.047$ & $-0.084,0.193$ \\
\hline
\end{tabular}

Note: Reported values at $p$-thresholds where nominally significant GxE effects were detected

"Sample where nominally significant GxE was detected. SLE categories (number of SLE reported): 0 SLE = "none", 1 or 2 SLE = "low", and 3 or more SLE = "high". In italic, nominally significant effects. In bold, robustly significant effect after conservative Bonferroni correction $\left(p<6.94 \times 10^{-4}\right)$

Between-sex differences in stress response could help to explain previous differences seen between sexes in depression such as those in associated risk (i.e., approximately 1.5-2-fold higher in women), symptoms reported and/or coping strategies (e.g., whereas women tend to cope through verbal and emotional strategies, men tend to cope by doing sport and consuming alcohol) ${ }^{63-67}$. This also aligns with an increased risk associated with a lack of social support seen in women compared to men $^{23}$. Furthermore, although we do not know whether participants experienced recent events with positive effects, we saw a protective effect in those women who did not experienced recent SLE $(p=0.037)$, suggesting that some genetic variants associated with MDD may operate as "plasticity alleles" and not just as "risk alleles" ${ }^{" 2,53}$. This effect was neutralized in the full cohort due to an opposite effect in men $(p=0.016)$, but it is supported by previous protective effects reported when using a serotoninergic multilocus profile score and absence of SLE in young women ${ }^{68}$. These findings would be consistent with a differentialsusceptibility model of depression ${ }^{69,70}$, also suggested by the interaction effects seen between the serotonin transporter linked promoter region gene (5-HTTLPR) locus and family support and liability to adolescent depression in boys ${ }^{71}$. However, our results and the examples given are only nominally significant and will require replication in larger samples. Robust identification of sex-specific differences in genetic stress-response could improve personalized treatments and therapies such as better coping strategies.

There are notable differences between our study and Colodro-Conde et al. to consider before accepting our findings as a replication of their results. First, differences in PRS profiling may have affected replication power. We used the same equivalent PGC-MDD2 GWAS as discovery sample. However, whereas Colodro-Conde et al. generated PRS in their target sample containing over $9.5 \mathrm{M}$ imputed SNP, in this study we generated PRS in a target sample of over $560 \mathrm{~K}$ genotyped SNPs (see Supplementary table 1 for comparison). This potentially results in a less informative PRS in our study, with less predictive power, although the variance explained by our PRS was slightly larger $(0.64 \%$ vs. $0.46 \%$ ). The size of the discovery sample is key to constructing an accurate predictive PRS, but to exploit the greatest number of the available variants may be an asset ${ }^{54}$. Secondly, different screening tools were used to measure both current depressive symptoms and recent environmental stressors across the two studies. Both studies transformed their data, using item response theory or by log-transformation, to improve the data distribution. 
However, neither study used depression scores that were normally distributed. The scale of the instruments used and their corresponding parameterization when testing for an interaction could have a direct effect on the size and significance of their interaction; ${ }^{55,72}$ so findings from GxE must be taken with caution. Furthermore, although both screening methods have been validated and applied to detect depressive symptoms, different questions may cover and emphasize different features of the illness, which may result in different results. The same applies to the measurement of environmental stressors in the two studies. Both covering of a longer time-period and upweighting by "dependent" SLE items may explain the increased explanatory power of "personal" SLE (12.9\%) in Colodro-Conde et al. to predict depression score compared to our "total" SLE measure (4.91\%). Finally, the unmeasured aspects of the exposure to SLE or its impact may also contribute to the lack of a stronger replication and positive findings.

In conclusion, despite differences in the measures used across studies, we saw concordance and similar patterns between our results and those of Colodro-Conde et al. ${ }^{23}$ Our findings, therefore, add validity to the diathesis-stress theory for depression. Empirically demonstrating the diathesis-stress theory for depression would validate recent ${ }^{20-22}$ and future studies using a genome-wide approach to identify genetic mechanisms and interactive pathways involved in GxE underpinning the causative effect of "stress" in the development of depressive symptoms and in mental illness in general. This study adds to our understanding of gene-by-environment interactions, although larger samples will be required to confirm differences in diathesis-stress effects between women and men.

\section{Acknowledgements}

A.A.S. is funded by the University of Edinburgh (www.ed.ac.uk) and MRC for his PhD study at the University of Edinburgh Institute of Genetics and Molecular Medicine (www.ed.ac.uk/igmm). D.J.M. acknowledges the financial support of NHS Research Scotland (NRS) through NHS Lothian. MA is supported by STRADL through a Wellcome Trust Strategic Award (reference 104036/Z/14/Z). Generation Scotland received core support from the Chief Scientist Office of the Scottish Government Health Directorates [CZD/16/6] and the Scottish Funding Council [HR03006]. The genotyping of the GS:SFHS samples was carried out by the Genetics Core Laboratory at the Wellcome Trust Clinical Research Facility, Edinburgh, Scotland and was funded by the Medical Research Council UK and the Wellcome Trust (Wellcome Trust Strategic Award "STratifying Resilience and Depression Longitudinally" (STRADL) Reference 104036/Z/14/Z). The Major Depressive Disorder Working Group of the Psychiatric Genomics Consortium depends on the contributions of many parties.

\footnotetext{
Author details

${ }^{1}$ Medical Genetics Section, Centre for Genomic and Experimental Medicine and MRC Institute of Genetics and Molecular Medicine, University of Edinburgh, Edinburgh, UK. ²Division of Psychiatry, Deanery of Clinical Sciences, Royal Edinburgh Hospital, University of Edinburgh, Morningside Park, Edinburgh EH10 5HF, UK. ${ }^{3}$ Health Informatics Centre, University of Dundee, Dundee, UK. ${ }^{4}$ Medical Research Council Human Genetics Unit, Institute of Genetics and Molecular Medicine, University of Edinburgh, Edinburgh, UK. ${ }^{5}$ Centre for Cognitive Ageing and Cognitive Epidemiology, University of Edinburgh, Edinburgh, UK
}

Conflict of interest

The authors declare that they have no conflict of interest.

\section{Publisher's note}

Springer Nature remains neutral with regard to jurisdictional claims in published maps and institutional affiliations.

Supplementary Information accompanies this paper at (https://doi.org/ 10.1038/541398-018-0356-7).

Received: 5 April 2018 Revised: 28 November 2018 Accepted: 10 December 2018

Published online: 18 January 2019

\section{References}

1. Hammen, C. Stress and depression. Annu Rev. Clin. Psychol. 1, 293-319 (2005).

2. Kessler, R. C. The effects of stressful life events on depression. Annu Rev. Psychol. 48, 191-214 (1997).

3. Kendler, K. S., Karkowski, L. M. \& Prescott, C. A. Causal relationship between stressful life events and the onset of major depression. Am. J. Psychiatry 156, 837-841 (1999).

4. Paykel, E. S. Life events and affective disorders. Acta Psychiatr. Scand 108, 61-66 (2003).

5. Stroud, C. B., Davila, J. \& Moyer, A. The relationship between stress and depression in first onsets versus recurrences: a meta-analytic review. J. Abnorm. Psychol. 117, 206-213 (2008).

6. Ensel, W. M., Peek, M. K. Lin, N. \& Lai, G. Stress in the life course: a life history approach. J. Aging Health 8, 389-416 (1996).

7. Kendler, K. S., Karkowski, L. M. \& Prescott, C. A. Stressful life events and major depression: risk period, long-term contextual threat, and diagnostic specificity. J. Nerv. Ment. Dis. 186, 661-669 (1998).

8. Mazure, C. M. Life stressors as risk factors in depression. Clin. Psychol. 5, 291-313 (1998).

9. Lichtenberg, P. \& Belmaker, R. H. Subtyping major depressive disorder. Psychother. Psychosom. 79, 131-135 (2010).

10. Elisei, S., Sciarma, T., Verdolini, N. \& Anastasi, S. Resilience and depressive disorders. Psychiatr. Danub. 25(Suppl 2), S263-S267 (2013).

11. Monroe, S. M. \& Simons, A. D. Diathesis-stress theories in the context of life stress research: implications for the depressive disorders. Psychol. Bull. 110 406-425 (1991).

12. Vogel, F. Schizophrenia genesis: the origins of madness. Am. J. Human. Genet. 48, 1218-1218 (1991).

13. Mann, J. J., Waternaux, C., Haas, G. L. \& Malone, K. M. Toward a clinical model of suicidal behavior in psychiatric patients. Am. J. Psychiatry 156, 181-189 (1999).

14. Riemann, D. et al. The hyperarousal model of insomnia: a review of the concept and its evidence. Sleep. Med Rev. 14, 19-31 (2010).

15. Bolt, M. A., Helming, L. M. \& Tintle, N. L. The associations between self-reported exposure to the chernobyl nuclear disaster zone and mental health disorders in Ukraine. Front. Psychiatry 9, 32 (2018).

16. Wray, N. R. \& Sullivan, P. F. Genome-wide association analyses identify 44 risk variants and refine the genetic architecture of major depression. Nature Genetics 50, 668-681 (2018).

17. Peyrot, W. J. et al. Effect of polygenic risk scores on depression in childhood trauma. Br. J. Psychiatry 205, 113-119 (2014).

18. Musliner, K. L. et al. Polygenic risk, stressful life events and depressive symptoms in older adults: a polygenic score analysis. Psychol. Med. 45, 1709-1720 (2015).

19. Peyrot, W. J. et al. Does childhood trauma moderate polygenic risk for depression? A Meta-analysis of 5765 Subjects From the Psychiatric Genomics Consortium. Biol. Psychiatry 84, 138-147 (2018).

20. Dunn, E. C. et al. Genome-Wide Association Study (GWAS) and Genome-Wide by Environment Interaction Study (GWEIS) of Depressive Symptoms in African American and Hispanic/Latina Women. Depress. Anxiety 33, 265-280 (2016).

21. Otowa, T. et al. The first pilot genome-wide gene-environment study of depression in the Japanese Population. PLoS One 11, e0160823 (2016).

22. Ikeda, M. et al. Genome-wide environment interaction between depressive state and stressful life events. J. Clin. Psychiatry 77, e29-e30 (2016). 
23. Colodro-Conde, L. et al. A direct test of the diathesis-stress model for depression. Mol. Psychiatry 23, 1590-1596 (2018).

24. Mullins, N. et al. Polygenic interactions with environmental adversity in the aetiology of major depressive disorder. Psychol. Med 46, 759-770 (2016).

25. lyegbe, C., Campbell, D., Butler, A., Ajnakina, O. \& Sham, P. The emerging molecular architecture of schizophrenia, polygenic risk scores and the clinical implications for GxE research. Soc. Psychiatry Psychiatr. Epidemiol. 49, 169-182 (2014).

26. McGrath, J. J., Mortensen, P. B., Visscher, P. M. \& Wray, N. R. Where GWAS and epidemiology meet: opportunities for the simultaneous study of genetic and environmental risk factors in schizophrenia. Schizophr. Bull. 39, 955-959 (2013).

27. Plomin, R. Commentary: missing heritability, polygenic scores, and geneenvironment correlation. J. Child Psychol. Psychiatry 54, 1147-1149 (2013).

28. Wray, N. R. et al. Research review: polygenic methods and their application to psychiatric traits. J. Child Psychol. Psychiatry 55, 1068-1087 (2014).

29. Smith, B. H. et al. Cohort Profile: Generation Scotland: Scottish Family Health Study (GS:SFHS). The study, its participants and their potential for genetic research on health and illness. Int J. Epidemiol. 42, 689-700 (2013).

30. Gunderson, K. L. Whole-genome genotyping on bead arrays. In DNA Microarrays for Biomedical Research: Methods and Protocols (ed. Dufva, M.) 197-213 (Humana Press, Totowa, 2009).

31. Kerr, S. M. et al. Pedigree and genotyping quality analyses of over 10,000 DNA samples from the Generation Scotland: Scottish Family Health Study. BMC Med. Genet. 14, 38 (2013).

32. Nagy, R. et al. Exploration of haplotype research consortium imputation for genome-wide association studies in 20,032 Generation Scotland participants. Genome Med. 9, 23 (2017).

33. Navrady, L. B. et al. Cohort Profile: Stratifying Resilience and Depression Longitudinally (STRADL): a questionnaire follow-up of Generation Scotland: Scottish Family Health Study (GS:SFHS). Int. J. Epidemiol 47, 13-14g (2018).

34. Purcell, S. et al. PLINK: a tool set for whole-genome association and population-based linkage analyses. Am. J. Hum. Genet 81, 559-575 (2007).

35. Smith, B. H. et al. Generation Scotland: the Scottish Family Health Study; a new resource for researching genes and heritability. BMC Med. Genet. 7, 74 (2006).

36. Fernandez-Pujals, A. M. et al. Epidemiology and Heritability of Major Depressive Disorder, Stratified by Age of Onset, Sex, and Illness Course in Generation Scotland: Scottish Family Health Study (GS:SFHS). PLoS One 10, e0142197 (2015).

37. Amador, C. et al. Recent genomic heritage in Scotland. BMC Genom. 16, 437 (2015).

38. Goldberg, D. P. \& Hillier, V. F. A scaled version of the General Health Questionnaire. Psychol. Med. 9, 139-145 (1979).

39. Sterling, M. General Health Questionnaire - 28 (GHQ-28). J. Physiother. 57, 259 (2011).

40. Goldberg, D. P. et al. The validity of two versions of the GHQ in the WHO study of mental illness in general health care. Psychol. Med. 27, 191-197 (1997).

41. Banks, M. H. Validation of the General Health Questionnaire in a young community sample. Psychol. Med. 13, 349-353 (1983).

42. Marks, A. D. G., Horrocks, K. A. \& Schutte, N. S. Emotional intelligence mediates the relationship between insecure attachment and subjective health outcomes. Personal. Individ. Differ. 98, 188-192 (2016).

43. O'Rourke, S., MacHale, S., Signorini, D. \& Dennis, M. Detecting psychiatric morbidity after stroke: comparison of the GHQ and the HAD Scale. Stroke $\mathbf{2 9}$ 980-985 (1998).

44. Kessler, R. C., Andrews, G., Mroczek, D., Ustun, B. \& Wittchen, H.-U. The World Health Organization Composite International Diagnostic Interview short-form (CIDI-SF). Int. J. Methods Psychiatr. Res. 7, 171-185 (1998).

45. Brugha, T., Bebbington, P., Tennant, C. \& Hurry, J. The List of Threatening Experiences: a subset of 12 life event categories with considerable long-term contextual threat. Psychol. Med. 15, 189-194 (1985).

46. Brugha, T. S. \& Cragg, D. The list of threatening experiences: the reliability and validity of a brief life events questionnaire. Acta Psychiatr. Scand. 82, 77-81 (1990).

47. Motrico, E. et al. Psychometric properties of the List of Threatening Experiences--LTE and its association with psychosocial factors and mental disorders according to different scoring methods. J. Affect Disord. 150 931-940 (2013).

48. Kendler, K. S., Karkowski, L. M. \& Prescott, C. A. The assessment of dependence in the study of stressful life events: validation using a twin design. Psychol. Med. 29, 1455-1460 (1999).

49. Euesden, J., Lewis, C. M. \& O'Reilly, P. F. PRSice: polygenic risk score software. Bioinformatics 31, 1466-1468 (2015).

50. Yang, J., Lee, S. H., Goddard, M. E. \& Visscher, P. M. GCTA: a tool for genomewide complex trait analysis. Am. J. Hum. Genet 88, 76-82 (2011).

51. Keller, M. C. Gene x environment interaction studies have not properly controlled for potential confounders: the problem and the (simple) solution. Biol. Psychiatry 75, 18-24 (2014).

52. Belsky, J. \& Beaver, K. M. Cumulative-genetic plasticity, parenting and adolescent self-regulation. J. Child Psychol. Psychiatry 52, 619-626 (2011).

53. Belsky, J. et al. Vulnerability genes or plasticity genes? Mol. Psychiatry 14, 746-754 (2009).

54. Dudbridge, F. Power and predictive accuracy of polygenic risk scores. PLoS Genet 9, e1003348 (2013).

55. Eaves, L. J., Last, K., Martin, N. G. \& Jinks, J. L. A progressive approach to nonadditivity and genotype-environmental covariance in the analysis of human differences. Br. J. Math. Stat. Psychol. 30, 1-42 (1977).

56. Polderman, T. J. et al. Meta-analysis of the heritability of human traits based on fifty years of twin studies. Nat. Genet. 47, 702-709 (2015).

57. Clarke, T. et al. Genetic and environmental determinants of stressful life events and their overlap with depression and neuroticism [version 2; referees: 3 approved with reservations]. Wellcome Open Res 3, 11 (2019).

58. Kendler, K. S., Kuhn, J. \& Prescott, C. A. The interrelationship of neuroticism, sex, and stressful life events in the prediction of episodes of major depression. Am. J. Psychiatry 161, 631-636 (2004).

59. Kendler, K. S. \& Eaves, L. J. Models for the joint effect of genotype and environment on liability to psychiatric illness. Am. J. Psychiatry 143, 279-289 (1986).

60. Plomin, R., DeFries, J. C. \& Loehlin, J. C. Genotype-environment interaction and correlation in the analysis of human behavior. Psychol. Bull. 84, 309-322 (1977).

61. Plomin, R, Lichtenstein, P, Pedersen, N. L, McClearn, G. E \& Nesselroade, J. R. Genetic influence on life events during the last half of the life span. Psychol. Aging 5, 25-30 (1990).

62. Arnau Soler, A. et al. Genome-wide by environment interaction studies (GWEIS) of depressive symptoms and psychosocial stress in UK Biobank and Generation Scotland. Transl. Psychiatry (in press).

63. Weissman, M. M. et al. Sex differences in rates of depression: cross-national perspectives. J. Affect Disord. 29, 77-84 (1993).

64. Van de Velde, S., Bracke, P. \& Levecque, K. Gender differences in depression in 23 European countries. Cross-national variation in the gender gap in depression. Soc. Sci. Med. 71, 305-313 (2010).

65. Labonte, B. et al. Sex-specific transcriptional signatures in human depression. Nat. Med 24, 525 (2018).

66. Angst, J. et al. Gender differences in depression. Epidemiological findings from the European DEPRES I and II studies. Eur. Arch. Psychiatry Clin. Neurosci. 252, 201-209 (2002).

67. Piccinelli, M. \& Wilkinson, G. Gender differences in depression. Crit. Rev. Br. J. Psychiatry 177, 486-492 (2000).

68. Vrshek-Schallhorn, S. et al. Additive genetic risk from five serotonin system polymorphisms interacts with interpersonal stress to predict depression. J. Abnorm. Psychol. 124, 776-790 (2015).

69. Belsky, J. \& Pluess, M. Beyond diathesis stress: differential susceptibility to environmental influences. Psychol. Bull. 135, 885-908 (2009).

70. Belsky, J., Bakermans-Kranenburg, M. J. \& van ljzendoorn, M. H. For better and for worse: differential susceptibility to environmental influences. Curr. Dir. Psychol. Sci. 16, 300-304 (2007).

71. Li, J. J., Berk, M. S. \& Lee, S. S. Differential susceptibility in longitudinal models of gene-environment interaction for adolescent depression. Dev. Psychopathol. 25, 991-1003 (2013).

72. Kang, S.-M. \& Waller, N. G. Moderated multiple regression, spurious interaction effects, and IRT. Applied Psychological Measurement 29, 87-105 (2005). 